\title{
A Case of Successful Recovery from High Dose Intravenous Nicorandil Infusion in Refractory Coronary Vasospasm with Hemodynamic Collapse
}

\author{
Won-Jun Koh, Jeong-Hyeon Cho, Ji-Hyun Lee, Won-Sik Kang, \\ Min-Kyung Lee, Jun-Hyoung Kim, Deok-Kyu Cho
}

Division of Cardiology, Cardiovascular Center, Myongji Hospital, Kwandong University College of Medicine, Goyang, Korea

A 70-year-old male came to the emergency room of the authors' hospital because of sudden cardiac arrest due to inferior wall ST elevation myocardial infarction. His coronary angiography revealed multiple severe coronary spasms in his very long left anterior descending artery. After an injection of intracoronary nitroglycerine, his stenosis improved. The cardiac arrest relapsed, however, accompanied by ST elevation of the inferior leads, while the patient was on diltiazem and nitrate medication to prevent coronary spasm. Recovery was not achieved even with cardiac massage, intravenous injection of epinephrine and atropine, and intravenous infusion of nitroglycerine. The patient eventually recovered through high-dose nicorandil intravenous infusion without ST elevation of his inferior leads. Therefore, intravenous infusion of a high dose of nicorandil must be considered a treatment option for cardiac arrest caused by refractory coronary vasospasm.

Key Words: Coronary vasospasm, Cardiopulmonary resuscitation, Nicorandil

\section{INTRODUCTION}

Coronary vasospasm causing acute myocardial infarction has been reported. ${ }^{1}$ Refractory coronary vasospasm has been relieved by intracoronary administration of nicorandil. ${ }^{2}$ This case reports how resuscitation was achieved through high dose intravenous nicorandil infusion in a cardiac arrest caused by refractory coronary vasospasm.

\section{CASE}

A 70-year-old man came to the hospital for acute chest pain that was accompanied by coma state and cyanosis. The patient had received treatment for high blood pressure and diabetes for the past 10 years. The patient was transported to

Received: August 20, 2012, Accepted: September 15, 2012

Corresponding Author: Deok-Kyu Cho, 697-24 Hwajungdong, Deokyang-gu, Division of Cardiology, Cardiovascular Center, Myongji Hospital, Kwandong University College of Medicine, Goyang 412-270, Korea Tel: (031) 810-6770, Fax: (031) 810-6778 E-mail: chodk123@paran.com the hospital wearing an oxygen mask. An automatic external defibrillator was used when the patient's heart rate slowed and cardiopulmonary resuscitation was performed for 6 minutes for asystole before the patient arrived at the emergency room. Blood pressure, pulse, and breathing were not measured and the patient was unconscious with a body temperature of $36.8^{\circ} \mathrm{C}$.

The serum biochemical examination, fasting glucose was $452 \mathrm{mg} / \mathrm{dL}$, creatinine was $1.6 \mathrm{mg} / \mathrm{dL}$, total cholesterol was $138 \mathrm{mg} / \mathrm{dL}$, triglyceride was $100 \mathrm{mg} / \mathrm{dL}$, high-density lipoprotein cholesterol was $36 \mathrm{mg} / \mathrm{dL}$, and low-density lipoprotein cholesterol was $85 \mathrm{mg} / \mathrm{dL}$. A pulse was perceived after cardiopulmonary resuscitation was performed for 8 minutes. An electrocardiogram (ECG) revealed ST elevation of the inferior leads and atrial fibrillation with rapid ventricular response (Fig. 1). The patient was diagnosed with acute myocardial infarction of the inferior wall and coronary angiography was performed as an emergency procedure.

The coronary angiography revealed that the right coronary artery was diminutive (Fig. 2A), the left anterior descending artery (LAD) was very long, and the inferior territory was fed by the LAD. More than 95\% stenosis accompanying the severe spasm was observed in the middle portion of the LAD (Fig. 2B), and near-total occlusion was verified in the far distal 


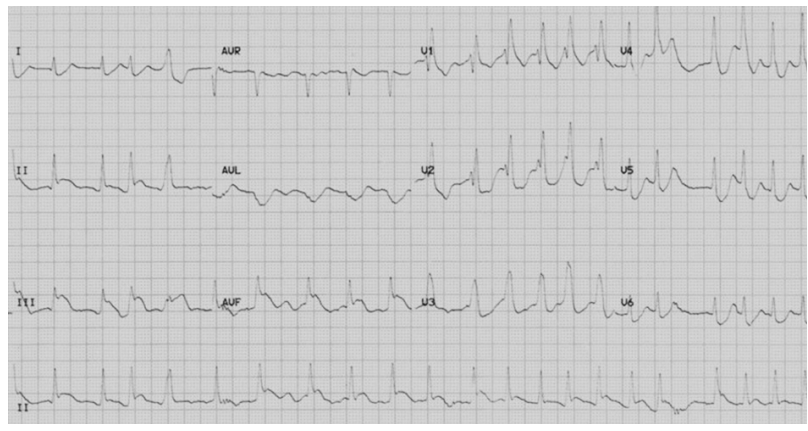

Fig. 1. The ECG after returning to spontaneous circulation through cardiopulmonary resuscitation. The ECG represented atrial fibrillation with rapid ventricular response and ST elevation in leads II, III, and aVF.
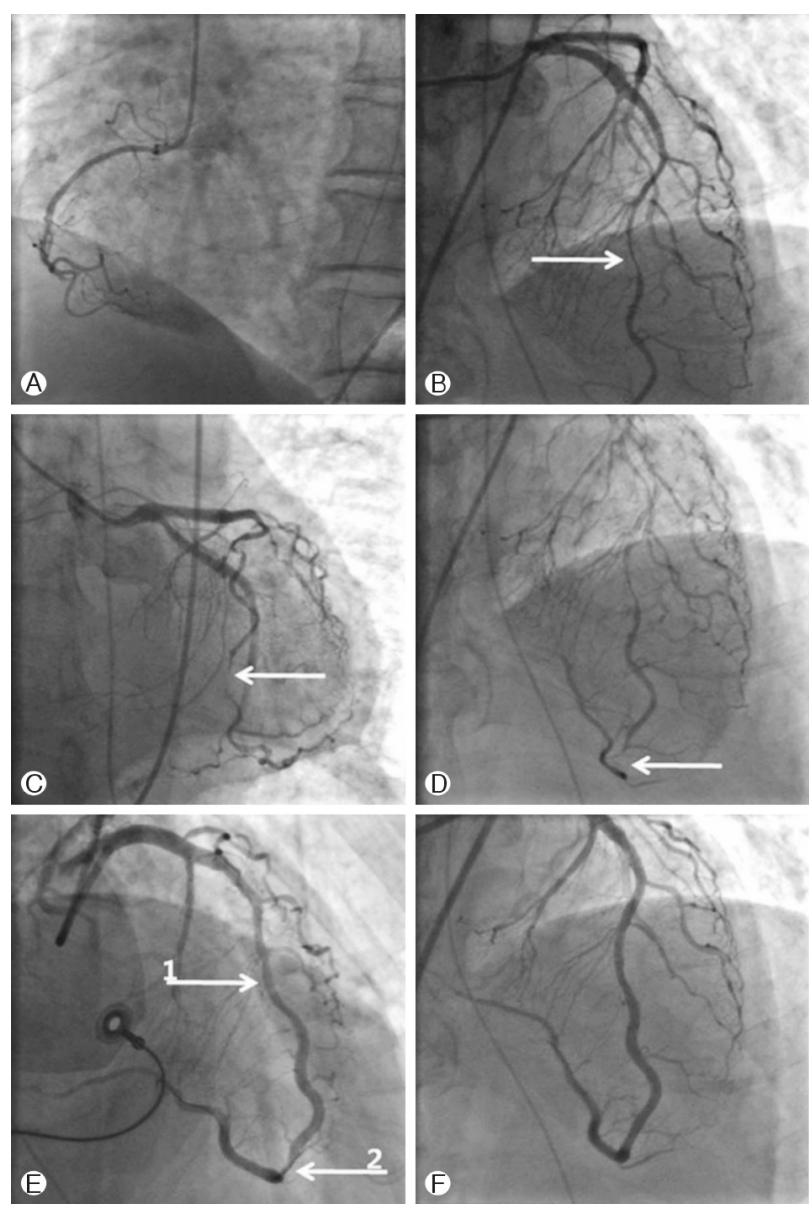

Fig. 2. The data on the coronary angiography. (A) Right coronary artery was diminutive. (B) More than 95\% stenosis accompanying severe spasm was evident in the middle portion of the LAD (white arrow). (C) and (D) Near total occlusion in the far distal portion of the LAD (white arrow). (E) The middle portion of the LAD showed improvement (white arrow 1), whereas the far distal portion continued to show stenosis. (white arrow 2) (F) Results after performing stenting in the distal portion of the LAD.

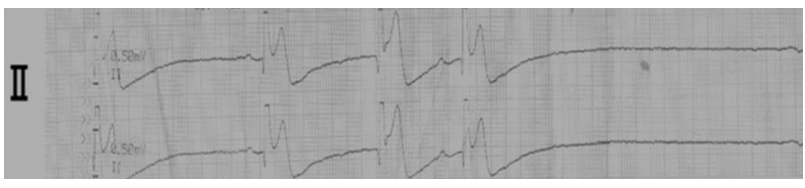

Fig. 3. Asystole observed after ST elevation in lead II.

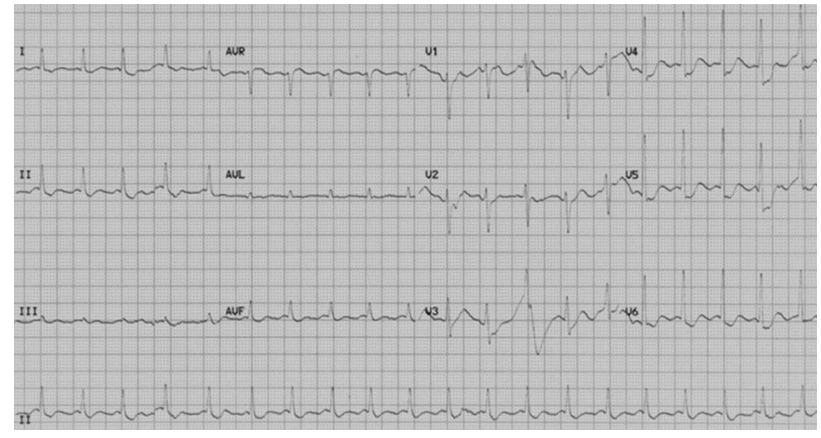

Fig. 4. The ST elevation of the inferior leads was no longer evident in the ECG after intravenous infusion of $48 \mathrm{mg}$ nicorandil.

portion of the $\mathrm{LAD}$ (Fig. 2C, 2D). After a $10 \mathrm{mg}$ injection of intracoronary nitroglycerine, stenosis in the middle portion of the LAD was recovered with $30 \%$ residual stenosis. But, improvement of the far distal portion of the LAD was not evident, where $90 \%$ stenosis was observed (Fig. 2E). After performing percutaneous transluminal coronary angiography using a $2.50 \times 20 \mathrm{~mm}$ coronary balloon (Amadeus ${ }^{\circledR}$; Eurocor Gmbh, Bonn, Germany), a $3.00 \times 20$ mm stent (Promus Element ${ }^{\circledR}$; Boston Scientific, Natick, MA, USA) was deployed to achieve successful revascularization (Fig. 2F).

The vital signs remained stable, and diltiazem $180 \mathrm{mg}$ and isosorbide dinitrate $80 \mathrm{mg}$ were administered to prevent coronary spasm. On the second day of hospitalization, the patient's heart rate began to slow down to $<40$ beats per minute, showing 2-3 mm ST elevation in lead II (Fig. 3). Asystole was considered to have caused the cardiac arrest, and cardiopulmonary resuscitation was performed. Atropine $1 \mathrm{mg}$ and epinephrine $1 \mathrm{mg}$ were administered three times at an interval of 3 minutes, and nitroglycerine $50 \mathrm{mg}$ was administered once through intravenous infusion. The patient did not recover from asystole. As the patient did not show improvement even through 10 minutes of cardiopulmonary resuscitation, nicorandil $48 \mathrm{mg}$ and normal saline $100 \mathrm{~mL}$ were mixed together and intravenous infusion was done for 3 minutes. The ST elevation in the inferior leads was no longer seen in the ECG and successful recovery was achieved (Fig. 4). Nicorandil was administered and the patient was stably maintained without additional coronary vasospasm. 


\section{DISCUSSION}

Coronary spasm is the most frequent cause of cardiac arrest in cardiac arrest survivors with no apparent heart disease. ${ }^{3}$ Coronary artery spasm plays an important role in the pathogenesis of a wide variety of ischemic heart diseases, including sudden cardiac death and, thus, could be one of the most impor tant functional abnormalities of the coronary artery. ${ }^{1,4}$ There are reports of coronary vasospasm causing acute myocardial infarction.

Nicorandil has a potassium channel-opening effect that activates adenosine triphosphate-sensitive potassium channels and induces nitric oxide expression in the same fashion as nitrates, resulting in increased intracellular cyclic GMP. ${ }^{5-7}$ Nicorandil also has cardioprotective effects in the ischemic myocardium, as demonstrated by reduced myocardial necrosis and improvement of myocardial stunning following coronary artery reperfusion, which mimics ischemic preconditioning. ${ }^{8-10}$ Nitrates and calcium antagonists are usually effective for coronary vasospasm and most patients respond to these drugs. This case has described that refractory coronary vasospasm was only relieved by intracoronary administration of nicorandil. $^{2}$ In comparison with nitrates, nicorandil is more useful to the cardiogenic shock with coronary spasm, in terms of relatively less effect on blood pressure without nitrate tolerance. ${ }^{11}$

Although this patient was administered diltiazem and isosorbide dinitrate to prevent coronary vasospasm, cardiac arrest was generated due to refractory coronary vasospasm. The coronary vasospasm could have been worsened by the epinephrine used in the resuscitation. Nitroglycerine was intravenously nicorandil using an emergency coronary angiography was impossible to perform in this situation, intravenous infusion of high dose nicorandil was performed to recover the patient from cardiac arrest caused by refractory coronary vasospasm. High dose nicorandil intravenous infusion should be considered as a treatment option in cases where cardiac arrest is caused by refractory coronary vasospasm.

\section{REFERENCES}

1. Takagi Y, Yasuda S, Takahashi J, Takeda M, Nakayama M, Ito $\mathrm{K}$, et al. Importance of dual induction tests for coronary vasospasm and ventricular fibrillation in patients surviving out-of-hospital cardiac arrest. Circ J 2009;73:767-9.

2. Hayashi T, Ichikawa M, Iwata A, Nakata M, Lim YJ, Mishima M. Intracoronary nicorandil relieves multiple coronary vasospasm with hemodynamic collapse. Circ J 2008;72:327-30.

3. Igarashi Y, Tamura T, Suzuki K, Tanabe Y, Tamura M, Yamaguchi $\mathrm{T}$, et al. High prevalence of coronary artery spasm in survivors of cardiac arrest with no apparent heart disease. Jpn Heart 1992;33:653-63.

4. Chevalier P, Kirkorian G, Touboul P. Arrhythmic sudden cardiac death due to coronary artery spasm. Card Electrophysiol Rev 2002;6:104-6.

5. Grover GJ. Pharmacology of ATP-sensitive potassium channel openers in models of myocardial and reperfusion. Can J Physiol Phamarcol 1997;75:309-15.

6. Taira N. Nicorandil as a hybrid between nitrates and potassium channel activators. Am J Cardiol 1989;63:18J-24J.

7. Holzmann S. Cyclic GMP as possible mediator of coronary arterial relaxation by Nicorandil (SG75). J Cardiovasc Pharmacol 1983;5:364-70.

8. Iwakura K, Ito H, Okamura A, Koyama Y, Date M, Higuchi $\mathrm{Y}$, et al. Nicorandil treatment in patients with acute myocardial infarction: a meta-analysis. Circ J 2009;73:925-31.

9. Schultz JE, Yao Z, Cavero I, Gross GJ. Glibenclamide-induced blockade of ischemic preconditioning is time dependent in intact rat heart. Am J Physiol 1997;272:H2607-15.

10. Kim JH, Jeong MH, Lim SY, Lee SH, Lee YS, Yoon KH, et al. The clinical effects of nicorandil during percutaneous coronary intervention in patients with unstable angina. Korean Circ J 2004;34:258-64. Korean.

11. Tabone X, Funck-Brentano C, Billon N, Jaillon P. Comparison of tolerance to intravenous nitroglycerin during nicorandil and intermittent nitroglycerin patch in healthy volunteers. Clin Pharmacol Ther 1994;56:672-9. 